MINI-SYMPOSIUM

\title{
Aortic intramural haematoma: natural history and predictive factors for complications
}

\author{
C A Nienaber, B M Richartz, T Rehders, H Ince, M Petzsch
}

Heart 2004;90:372-374. doi: 10.1136/hrt.2003.027615

l tramural haematoma (IMH) of the aorta is attracting growing interest as a variant of aortic dissection and is more frequently diagnosed by modern tomographic imaging modalities in the evaluation of acute aortic syndromes. ${ }^{12}$ The evolution from IMH to overt dissection or even rupture may occur suddenly or is heralded by ongoing acute aortic (pain) syndrome. Unlike classic aortic dissection, IMH has no mechanisms of decompression by a re-entry tear but rather reveals intramural (intramedial) thickening or echolucent pockets of non-communicating blood with potential for rupture or, at times, regression and resorption of haematoma with time..$^{3-6}$ As in overt dissection, widening of the mediastinum or the aortic shadow, pleural effusion and pain, aortic regurgitation, and pericardial effusion may emerge after initial IMH, whereas focal neurological signs or malperfusion syndrome are incidental. ${ }^{7}$ Hence, the subtle initial pathology of IMH is more likely to be missed than overt dissection, especially in the absence of recurrent pain ("aortic syndrome"). While diagnostic and therapeutic implications of IMH continue to impact on vascular medicine, description of natural course and prediction of individual risk is far from settled. ${ }^{6-10}$

PREDICTORS OF EARLY PROGRESSION AND DEATH Observational data from independent groups revealed evidence that the IMH evolves to resorption or progression to either classic dissection, contained rupture or formation of an aneurysm within 30 days of hospital admission.

Proximal location of IMH is clearly considered an independent predictor of progression to dissection, contained rupture, or aneurysm formation. In our series of 66 cases of IMH ( 38 type A and 28 type B), 73\% of progressive IMH were type $\mathrm{A}$ as compared to $44 \%$ in stable IMH (odds ratio (OR) $4.3,95 \%$ confidence interval (CI) 1.5 to $12.3 ; \mathrm{p}=0.02$ ); early progression was unrelated to age, sex, chronic arterial hypertension, Marfan syndrome, bicuspid aortic valve, and both local extent and diameters at the site of IMH. ${ }^{10}$

Considering a 30 day mortality rate of $20 \%$ and a five year survival of $43 \%$, our findings are supported by the global experience in 456 cases of IMH with an early death rate of $16 \%$, whereas five year survival rates are not available. ${ }^{5}{ }^{11-13}$ Proximal (type A) IMH is no longer related to early death when surgical repair is performed ${ }^{2}{ }^{10}$ (fig l). The high risk of "wait and see" in type A IMH, though, is reflected in $55 \%$ early mortality with medical treatment compared to $8 \%$ with surgical repair $(p=0.004)$. Considering a $12 \%$ early mortality after surgery, and a $24 \%$ death rate with medical treatment, global experience from the International Registry of Aortic Dissection confirmed a trend to better outcome after surgery of proximal IMH $(\mathrm{p}=0.12)$.

Interestingly, Asian patients were quoted with low death rates in proximal IMH even without surgery. ${ }^{82-14}$ However, 10 of 22 patients with type A IMH (45\%) underwent surgical repair and four cases with medical management developed cardiac tamponade. ${ }^{4}$ Similarly, tamponade was observed in two of three patients with type A IMH surviving medical treatment. ${ }^{12}$ Nishigami and colleagues reported on eight medically treated type A IMH with seven survivors; yet IMH requiring surgery, and cases with aortic diameters of $>50 \mathrm{~mm}$ or cardiac tamponade were excluded from this analysis. Similarly, in Korean patients with type A IMH treated medically, only one of 18 patients died, but four patients required pericardiocentesis or surgery for proximal dissection. ${ }^{14}$ Finally, $54 \%$ of cases with aortic diameters $<50 \mathrm{~mm}$ eventually progressed to dissection or rupture, challenging the Asian experience that IMH in a normal sized aorta precludes progression. This finding is far from paradoxical considering that the aortic wall is thickened from intramural blood at the expense of luminal width. Thus, the Asian experience in fact underlines findings in the European gene pool that proximal IMH frequently leads to serious complications.

\section{PREDICTORS OF LATE PROGRESSION}

Late progression of IMH was associated with younger age (49 (17) years $v 64$ (11) years in IMH without late progression; $\mathrm{p}<0.003)$, Marfan syndrome $(\mathrm{p}=0.02)$, and absence of $\beta$ adrenergic antagonists (only 7\% of IMH with late progression were treated with $\beta$ blockers compared to $49 \%$ of IMH patients without late progression; $p=0.009)$. On multivariate analysis only younger age (OR $0.92,95 \% \mathrm{Cl} 0.86$ to $0.98 ; \mathrm{p}=0.01)$ and absence of long term oral $\beta$ blocker treatment (OR 10.8, 95\% Cl 1.2 to 96.4; $\mathrm{p}=0.03$ ) were confirmed as independent predictors of late progression (table 1, fig 2); Marfan syndrome failed to qualify as a risk predictor on multivariate analysis $(\mathrm{p}=0.5)$.

Actuarial survival analysis of IMH confirmed better long term outcome on oral $\beta$ blocker treatment (95\% $v 67 \%$ in patients without $\beta$ blocker treatment $(p=0.004)$. $\beta$ Adrenergic blocking agents are effective in managing classic aortic dissection and in retarding progression of aortic root

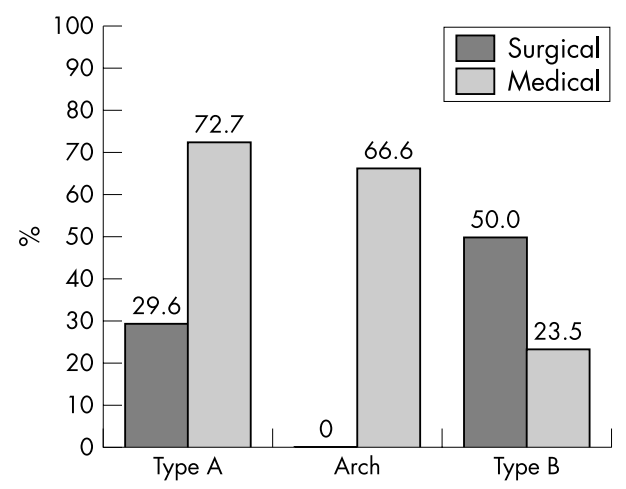

Figure 1 Mortality rates from intramural haematoma: overall mortality within one year in relation to medical or surgical treatment. 


\begin{tabular}{|c|c|c|c|}
\hline Variable & OR & $95 \% \mathrm{Cl}$ & $p$ Value \\
\hline \multicolumn{4}{|l|}{ Early progression } \\
\hline Type A IMH & 3.894 & 1.306 to 11.61 & 0.02 \\
\hline Aortic diameter (mm) & 0.958 & 0.922 to 0.997 & 0.03 \\
\hline \multicolumn{4}{|l|}{ Late progression } \\
\hline Absence of long term & & & \\
\hline$\beta$ blocker treatment & 10.8 & 1.2 to 96.4 & 0.03 \\
\hline Age $<55$ years & 0.92 & 0.86 to 0.98 & 0.01 \\
\hline
\end{tabular}

$\mathrm{Cl}$, confidence interval; IMH, intramural haematoma; $\mathrm{OR}$, odds ratio. Marfan syndrome was not significant on multivariate analysis $(p=0.5)$ and was thus not entered into the final model.

aneurysm in both Marfan syndrome and abdominal aneurysm. $\beta$ Blockers protect by reducing wall stress, systolic arterial pressure, and the rate of pressure changes and, presumably, by stabilising the extracellular vascular matrix of the aorta. ${ }^{15}$ Thus, our finding that effective $\beta$ blocker treatment infers a reduced rate of late progression is novel, ${ }^{10}$ but not unexpected in IMH considering the beneficial impact of vigorously lowering the driving force for dissection.

The observation that older age ( $>55$ years) at initial diagnosis of IMH has a better long term prognosis may be explained by more focal microscars along the aortic wall inherently limiting the longitudinal progression of $\mathrm{IMH}^{25}$ Accordingly, favourable outcomes of IMH are consistently reported in patients beyond 65 years. ${ }^{812}{ }^{13}$ Thus, considering both advanced aortosclerosis with older age and the lower risk of progression, a conservative strategy (with $\beta$ blockade and serial imaging) may be justified in elderly multimorbid patients and in distal IMH. ${ }^{10}$

\section{INTRAMURAL HAEMATOMA AND PENETRATING AORTIC ULCERS}

Ulcer-like projections in aortic segments of IMH identify a subset of patients at high risk. Penetrating atherosclerotic ulcers (PAU) are known to result from progressive erosion of atheromatose mural plaque penetrating the elastic lamina, finally separating media layers and setting the stage for adjacent intramural haematoma with either local or longitudinal progression. PAUs are preferentially (>90\%) observed in IMH of the descending aorta, while IMH without PAU is more frequently present in the ascending aorta.

Symptomatic PAU infers complications such as formation of aneurysm, pseudoaneurysm, and dissection, or unpredictable rupture. Careful imaging is vital to identify both diameter and depth of ulcers with $\mathrm{IMH}$, since width $>2 \mathrm{~cm}$ and depth $>1 \mathrm{~cm}$ may herald the need for interventional or surgical repair to avoid rupture and death. ${ }^{9}{ }^{10}$
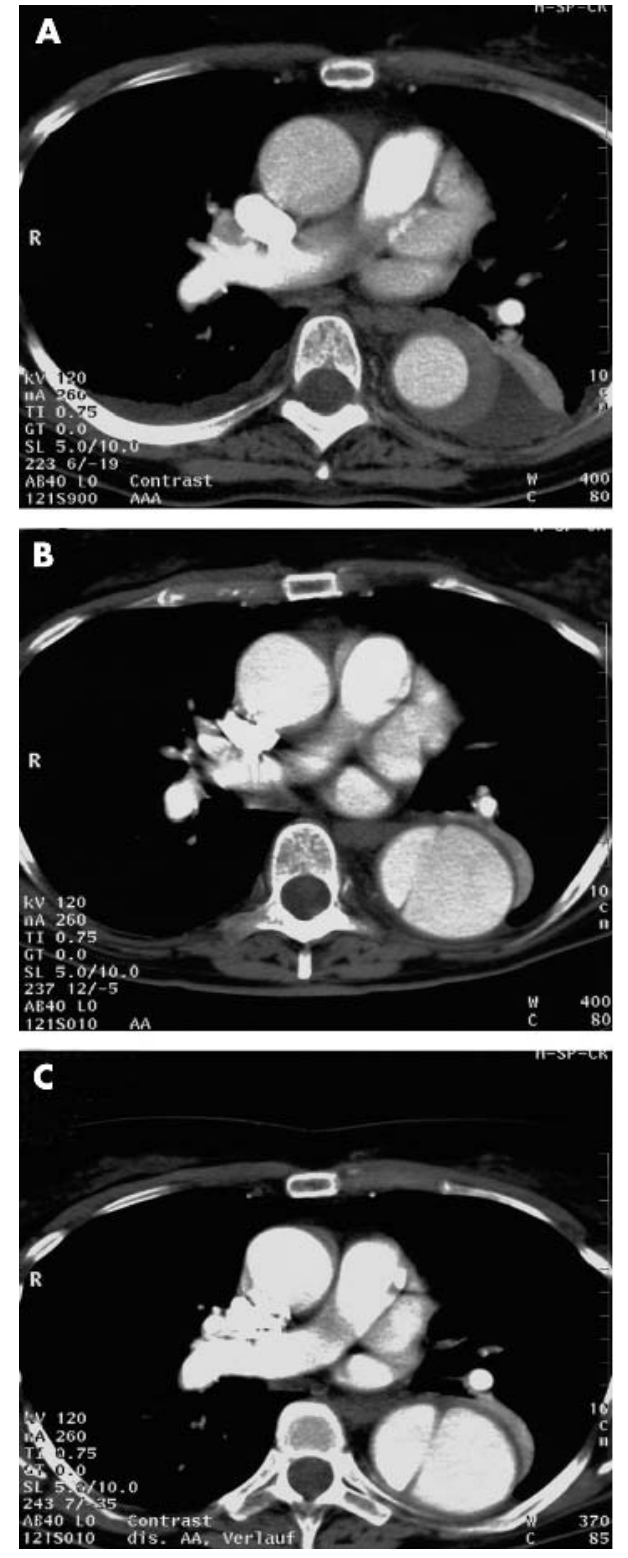

Figure 2 Contrast enhanced spiral computed tomography documenting the evolution of acute intramural haematoma of the descending aorta to open dissection with communication between the true and a false lumen: (A) baseline; (B), three months; (C) six months.

\section{SIGNS OF PROGRESSION IN INTRAMURAL HAEMATOMA}

Predictors of IMH progression are summarised in table 2. Persistent and recurrent pain despite aggressive medical

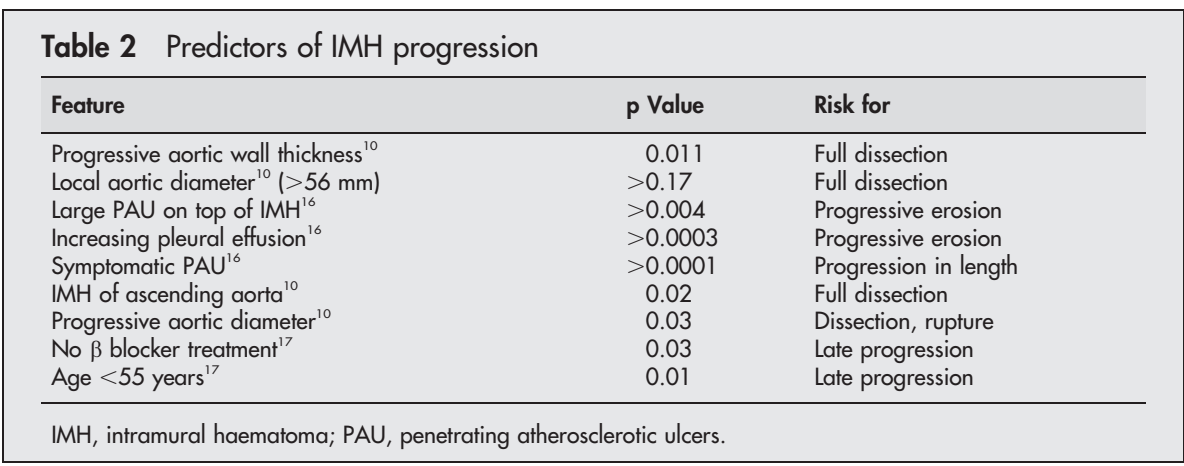


treatment, or repetitive pleural effusion, are important indicators of disease progression. ${ }^{9}$ While large PAU may be a common denominator of progression and adverse outcome, absence of PAU does not predict a stable course of IMH. A closer look reveals that $75 \%$ of proximal IMH patients died or had surgical replacement by the time of follow up. Conversely, IMH of the descending aorta may indeed warrant watchful waiting, supporting a previous notion. ${ }^{1268}$ Moreover, a recent meta-analysis on morbidity and mortality of both IMH and ulcerations of the aorta offers additional insight. ${ }^{5}{ }^{16}$ The natural course of 168 cases of typical IMH led to overt aortic dissection in $25 \%$ of ascending aortic IMH and in $13 \%$ of descending IMH, to aortic rupture in $28 \%$ and $9 \%$, respectively, or to stabilisation in $28 \%$ and $76 \%$, respectively; 30 day mortality was $18 \%$ with surgical repair of proximal $\mathrm{IMH}$, and 33\% with surgery to distal IMH compared to $60 \%$ and $8 \%$ with medical treatment of proximal and distal IMH, respectively.

IMH as "acute aortic syndrome" is indicative of a dynamic process and imminent events, and thus should focus our attention on diagnostic confirmation of progression with subsequent treatment by either surgical repair or interventional stent-graft placement. ${ }^{9}{ }^{10}$

\section{CONCLUSIONS}

IMH of the aorta is a potentially lethal disorder with frequent progression to aortic rupture, dissection or aneurysm. Short term prognosis is serious in IMH involving the ascending aorta, and surgical repair improves outcome regardless of the aortic diameter. IMH of the descending aorta, especially when confined to a short segment or with aortic diameters $<50 \mathrm{~mm}$ has a better outcome, but may not be excluded from early progression. Five year survival of IMH is $<50 \%$; yet late progression is neither predicted by location of IMH or width of the aorta nor by presence of risk factors. Long term prognosis, however, may benefit from chronic effective $\beta$ blockade regardless of surgical repair.

\section{ACKNOWLEDGEMENTS}

The authors are indebted to Mrs G Knoop and Mrs S Lehr for secretarial assistance and artwork.

\section{Authors' affiliations}

C A Nienaber, B M Richartz, T Rehders, H Ince, M Petzsch, Division of Cardiology at the University Hospital Rostock, Department of Internal Medicine, Rostock, Germany

Correspondence to: Christoph A Nienaber, MD, Division of Cardiology, University Hospital of Rostock, Ernst-Heydemann-Str. 6, 18057 Rostock, Germany; christoph.nienaber@med.uni-rostock.de

\section{REFERENCES}

1 Nienaber CA, Sievers $\mathrm{H}-\mathrm{H}$. Intramural hematoma in acute aortic syndromemore than one variant of dissection? Circulation 2002;106:284-5.

2 Nienaber CA, von Kodolitsch Y, Petersen B, et al. Intramural hemorrhage of the thoracic aorta: diagnostic and therapeutic implications. Circulation 1995:92:1465-72.

3 Ohmi M, Tabayashi K, Moizumi Y, et al. Extremely rapid regression of aortic intramural hematoma. J Thorac Cardiovasc Surg 1999;1 18:968-9.

4 Neri E, Capannini G, Carone E, et al. Evolution toward dissection of an intramural hematoma of the ascending aorta. Ann Thorac Surg 1999:68:1855-66.

5 von Kodolitsch Y, Nienaber CA. Intramural hemorrhage of the thoracic aorta: natural history, diagnostic and prognostic profiles of 209 cases with in vivo diagnosis. Z Kardiol 1998;87:797-807.

6 Robbins RC, McManus RP, Mitchell RS, et al. Management of patients with intramural hematoma of the thoracic aorta. Circulation 1993;88:1-10.

7 von Kodolitsch Y, Schwartz AG, Nienaber CA. Clinical prediction of acute aortic dissection. Arch Intern Med 2000;160:2977-82.

8 Kaji S, Nishigami K, Akasaka T, et al. Aortic and peripheral vascular surgery: prediction of progression or regression of type A aortic intramural hematoma by computed tomography. Circulation 1999;100:11-281-8.

9 Ganaha F, Miller DC, Sugimoto K, et al. The prognosis of aortic intramural hematoma with and without penetrating atherosclerotic ulcer: a clinical and radiological analysis. Circulation 2002;106:342-8.

10 von Kodolitsch Y, Csösz SK, Koschyk DH, et al. Intramural hematoma of the aorta-predictors of progression to dissection and rupture. Circulation 2003; 107: 11 158-63.

11 Harris KM, Bravermann AC, Gutierrez FR, et al. Transesophageal echocardiographic and clinical features of aortic intramural hematoma. J Thorac Cardiovasc Surg 1997;114:619-26.

12 Shimizu H, Yoshino H, Udagawa H, et al. Prognosis of aortic intramural hemorrhage compared with classic aortic dissection. Am J Cardiol 2000;85:792-5.

13 Nishigami K, Tsuchiya T, Shono $\mathrm{H}$, et al. Disappearance of aortic intramural hematoma and its significance to the prognosis. Circulation 2000; 102(suppl III):III-243-7.

14 Song JK, Kim HS, Kang DH, et al. Different clinical features of aortic intramural hematoma versus dissection involving the ascending aorta. J Am Coll Cardiol 2001;37:1604-10.

15 Shores J, Berger KR, Murphy EA, et al. Progression of aortic dilatation and the benefit of long-term beta-adrenergic blockade in Marfan syndrome. N Engl J Med 1994;330:1335-41.

16 von Kodolitsch Y, Nienaber CA. Penetrating ulcer of the thoracic aorta: natural history, diagnostic and prognostic profiles. Z Kardiol 1998;87:917-27.

\section{ELECTRONIC PAGES}

\section{Heart Online case reports: www.heartjnl.com}

$7 \mathrm{c}$ he following electronic only articles are published in conjunction with this issue of Heart.

\section{Malignant vasovagal syndrome in two patients with Wolff-Parkinson-White syndrome \\ N M Gandhi, D H Bennett}

The presence of Wolff-Parkinson-White (WPW) syndrome in patients presenting with syncope suggests that tachyarrhythmia may be the cause. However, the symptoms require careful evaluation. Two young patients presented with syncope and were found to have WPW syndrome on their ECG. In both patients symptoms were suggestive of vasovagal syncope. During tilt testing, both the patients developed their typical symptoms with a fall in blood pressure and heart rate confirming the diagnosis of malignant vasovagal syndrome.

(Heart 2004;90:e19) www.heartjnl.com/cgi/content/full/90/ $4 / \mathrm{e} 19$
Situs inversus and acute coronary syndrome M Saha, S Chalil, N Sulke

Dextrocardia is a rare clinical phenomenon with a reported incidence of one in 10000 . Consequently, acute coronary syndromes in such patients are rare. When chest pain occurs in this setting, it is important to be aware of the unique problems that may occur if coronary intervention is contemplated. Previous case reports have shown successful attempts at reperfusing one coronary artery percutaneously. In this case, coronary stents were successfully deployed in two vessels of a man with situs inversus, which has not been reported before. Additionally, the angiographic results that followed from successful intervention did not produce the expected haemodynamic and electrocardiographic changes.

(Heart 2004;90:e20) www.heartjnl.com/cgi/content/full/90/ $4 / \mathrm{e} 20$ 